\title{
Synthesis and anticancer evaluation of novel 3,5-diaryl-thiazolo[4,5-d]pyrimidin-2-one derivatives
}

\author{
Lilianna Becan $\cdot$ Edwin Wagner
}

Received: 24 January 2012/ Accepted: 11 September 2012/Published online: 4 October 2012

(C) The Author(s) 2012. This article is published with open access at Springerlink.com

\begin{abstract}
The 2-oxo analogs of thiazolo[4,5- $d]$ pyrimidine-2-thiones were prepared to study their cytotoxic activity. Five of the newly synthesized compounds were selected by the National Cancer Institute (Bethesda, MD, USA) for a primary in vitro antitumor assay. 7-Chloro-3,5diphenyl-thiazolo[4,5- $d$ ]pyrimidin-2-one (5a) proved to be the most active one among the screened derivatives and was further evaluated in the full panel of 60 cell lines at five different concentrations. The structures of compounds were determined by IR, ${ }^{1} \mathrm{H}-\mathrm{NMR},{ }^{13} \mathrm{C}-\mathrm{NMR}$, X-ray, and elemental analysis.
\end{abstract}

Keywords Antitumor agents - In vitro studies ·

Thiazolo[4,5- $d]$ pyrimidin-2-ones $\cdot$ Synthesis

\section{Introduction}

Thiazolo[4,5- $d]$ pyrimidines, 7-thio analogs of purines are potentially bioactive molecules. In contrast with related 2-thioxo-thiazolo[4,5-d]pyrimidine derivatives, the 2-oxo analogs have not been very well explored in medicinal chemistry. The synthesis and biological evaluation of the substituted 2-oxo-thiazolo[4,5- $d$ ] pyrimidines have been the subject of several review articles. They were reported to possess antibacterial, antifungal (Akbari et al., 2008; Habib et al., 2007), and anti-inflammatory activity (CXCR2receptor antagonists) (Walters et al., 2008), inhibit the growth of HCMV-human cytomegalovirus (Revankar et al., 1998), and be corticotrophin-releasing hormone (CRH-R1)

L. Becan $(\bowtie) \cdot$ E. Wagner

Department of Drugs Technology, Wroclaw Medical University,

Pl. Nankiera 1, 50-140 Wroclaw, Poland

e-mail: 1b1963@orange.pl receptor antagonists (display antidepressant activity) (Beck et al., 1999).

In this study, in continuation of our work on thiazolo[4,5d]pyrimidine derivatives, the synthesis and in vitro cytotoxic evaluation of thiazolo[4,5- $d]$ pyrimidin-2-ones are reported. These designed thiazolo[4,5- $d$ ] pyrimidine-2-ones are related to thiazolo $[4,5-d]$ pyrimidine-2-thiones that have been previously reported to be potent antitumor agents (Becan and Wagner, 2008). Thiazolo[4,5- $d]$ pyrimidine derivatives have been extensively studied as potential drug candidates and also have anticancer activity (Rida et al., 1996; Fahmy et al., 2002, 2003). Most of these compounds provided with anticancer activity possess an aromatic rings and electronegative substituent directly linked to the $\mathrm{C}-17$ of the essential core (Fig. 1) or attached at aromatic moieties. The method involved subsequent treatment of the appropriate 3,5-diaryl2-thioxo-5,6-dihydro- $4 H$-thiazolo[4,5- $d$ ]pyrimidin-7-ones (2) and 7-chloro-3,5-diaryl-thiazolo[4,5- $d]$ pyrimidine-2thiones $(3)$ with diethyl sulfate and water for the replacement of the 2-thioxo group by an oxygen function (Scheme 1). Compounds 2 and 3 were obtained from 4-amino-5-carboxamido-3-substituted-2,3-dihydrothiazole-2-thiones (1) (Gewald, 1966) according to a reported earlier procedure (Becan and Wagner, 2008). Pyrimidine ring formation with appropriate aryl aldehyde, followed by chlorination provided the desired cores $\mathbf{2}$ and $\mathbf{3}$, bearing the respective aromatic substituent at position 3 and 5 , which could further be treated with diethyl sulfate and hydrolyzed to yield 2-thiazolones 4a-4f and 5a-5f. All synthesized compounds were submitted to the National Cancer Institute (NCI, Bethesda, Maryland) to evaluate their growth inhibitory effects on 60 human cancer cell lines, derived from nine neoplasmatic diseases. Five derivatives $\mathbf{4 a}, \mathbf{4 b}, \mathbf{5 a}, \mathbf{5 b}$, and $\mathbf{5 d}$ were selected for a primary in vitro antitumor assay, at $10^{-5} \mathrm{M}$ concentration. Results were expressed as percent growth of 


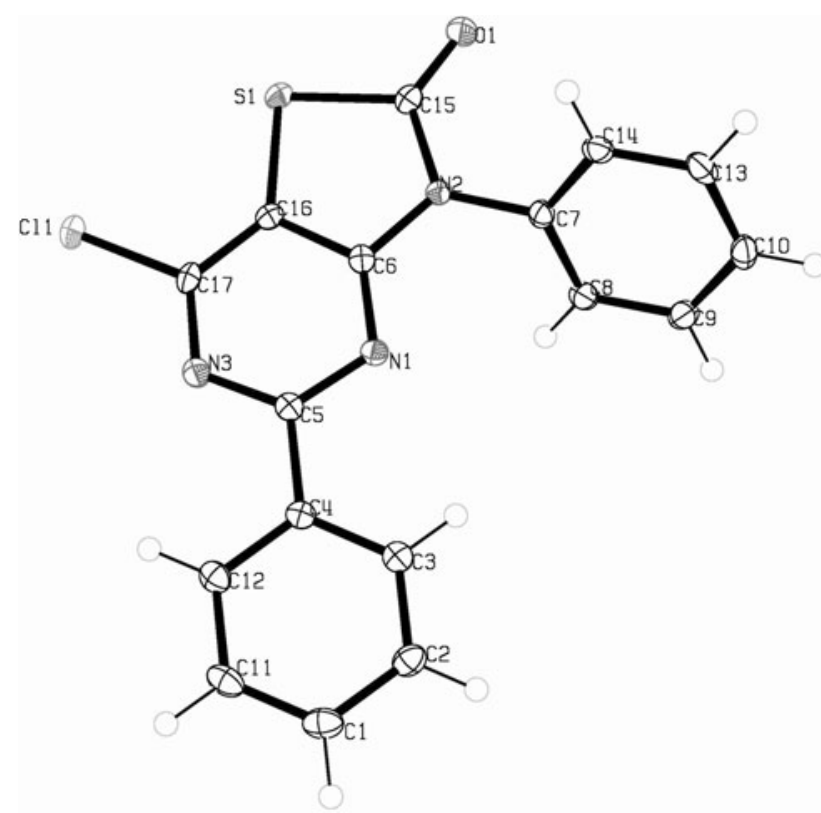

Fig. 1 X-Ray molecular structure of compound 5a with the atomnumbering scheme used in the crystallographic analysis

the treated cells, compound 5a showing mean percent growth $=71.26$ was further tested at five different concentrations.

\section{Results and discussion}

Chemistry

A series of the new substituted thiazolo[4,5- $d$ ]pyrimidin2-ones $\mathbf{4 a - 4 f}$ and $\mathbf{5 a - 5 f}$ were synthesized as shown in Scheme 1. The method involved subsequent treatment of the appropriate 3,5-diaryl-2-thioxo-5,6-dihydro-4H-thiazolo [4,5-d]pyrimidin-7-ones (2) and 7-chloro-3,5-diaryl-thiazolo[4,5- $d]$ pyrimidine-2-thiones (3) (Becan and Wagner, 2008) with diethyl sulfate and water for the replacement of the 2-thioxo group with 2-oxo. First, compounds 2 were obtained through the reaction of the corresponding, refluxing aromatic aldehyde with 4-amino-5-carboxamido-3-substituted-2,3-dihydrothiazole-2-thiones 1 (Gewald, 1966), in the presence of bases according to the earlier reported procedure (Becan and Wagner, 2008). Pyrimidine ring formation with aryl aldehydes followed by chlorination with a mixture of phosphorus pentachloride and phosphorus oxychloride gave the desired cores $\mathbf{2}$ and $\mathbf{3}$ which were further treated in boiling acetonitrile with diethyl sulfate. The obtained positively charged 2-ethyltiothiazolium salt was hydrolyzed to yield thiazolones-2. Yields of reaction were variable and were higher when $\mathrm{R} 1$ and $\mathrm{R} 2$ were not substituted. Elemental analysis, IR, ${ }^{1} \mathrm{H}$ and ${ }^{13} \mathrm{C}-\mathrm{NMR}$, and X-ray data evaluated the structure of synthesized substances. In the IR spectra of compounds $\mathbf{4 a - 4 f}$, the two stretching bands of 6-NH group were detected in the range of 3470-3080 $\mathrm{cm}^{-1}$. These compounds showed the characteristic vibrations of the $\mathrm{C}=\mathrm{O}$ group at $1690-1670 \mathrm{~cm}^{-1}$. In the ${ }^{1} \mathrm{H}-\mathrm{NMR}$ spectra, characteristic signal of compounds $\mathbf{4 a - 4 f}$ was one-proton singlet of $6 \mathrm{~N}-\mathrm{H}$ resonated at $13.19-13.27 \mathrm{ppm}$. Aromatic protons have formed multiplet at $7.22-8.20 \mathrm{ppm}$.

The formation of chlorination products $\mathbf{5 a}-\mathbf{5 f}$ was indicated in the IR spectra by the disappearance of stretching bands of 6-NH group. Besides the absorption bands due to $\mathrm{C}=\mathrm{N}$ and $\mathrm{C}-\mathrm{S}-\mathrm{C}$ functions, the presence of $\mathrm{C}=\mathrm{O}$ functional group was marked by the appearance of bond ranging from 1690 to $1680 \mathrm{~cm}^{-1}$, which was lacked in the precursor 3 . In the ${ }^{1} \mathrm{H}-\mathrm{NMR}$ spectra of 7 -chloro derivatives $\mathbf{5 a}-\mathbf{5 f}$ we were observed only aromatic protons signal at $7.26-8.22 \mathrm{ppm}$. The ${ }^{13} \mathrm{C}-\mathrm{NMR}$ spectra of the active compounds $\mathbf{5 a}, \mathbf{5 b}$, and $\mathbf{5 d}$, given in Table 1, displayed the appropriate number of resonances that exactly fit the number of carbon atoms. The most active compound 5a was recrystallized from a DMF solvent; the blockshaped crystals formed as a result were submitted to X-ray analysis. Data were collected at $100 \mathrm{~K}$ from a single crystal. X-ray crystallography of the most active agent 5a confirmed the chemical structure (Fig. 1). Crystallographic data for the structure are depicted in Table 2.

\section{Anticancer activity assay}

All synthesized compounds were submitted for testing at the NCI to evaluate the growth inhibitory effect. Five compounds $\mathbf{4 a}, \mathbf{4 b}, \mathbf{5 a}, \mathbf{5} \mathbf{b}$, and $\mathbf{5 d}$ were selected for a primary in vitro antitumor assay (Monks et al., 1991; Boyd and Paull, 1995; Shoemaker et al., 2002). A process beginning with the evaluation of the compound against approximately 60 different human tumor cell lines representing leukemia, melanoma, and cancers of the lung, colon, brain, breast, ovary, prostate, and kidney at $10^{-5} \mathrm{M}$ concentration was performed. With one dose, compound 4b was devoid of cytotoxic activity (mean growth percent 99.88) and 4a was slightly active against renal cancer CAKI-1 cell line (26.76\% growth).

Compounds 5a, 5b, and 5d which possess electronwithdrawing 7-chloro substituent showed variable antitumor activity, reported as the percentage of growth of treated cells; the preliminary screening results are shown in Table 3. Compounds 5a, 5b, and 5d exhibited antiproliferative effect against cell lines of leukemia, non-small cell lung cancer, colon cancer, melanoma, ovarian cancer, and renal cancer. It is worth to mention that substance 5a showed noticeable cytotoxic activity against renal cancer (UO-31), melanoma (MALME-3M), and non-small cell lung cancer (NCI-H522) while compound 5b was most 
<smiles>NC(=O)c1sc(=S)n(-c2ccc(-c3ccccc3)cc2)c1N</smiles><smiles>[R]c1ccc(-n2c(=S)sc3c(=O)[nH]c(-c4cccc([R2])c4)nc32)cc1</smiles><smiles>[R]c1ccc(-n2c(=S)sc3c(Cl)nc(-c4cccc([R2])c4)nc32)cc1</smiles><smiles>[R]c1ccc(-n2c(=O)sc3c(=O)[nH]c(-c4cccc([R])c4)nc32)cc1</smiles>

(iii)<smiles></smiles><smiles>[R17]c1ccc(-n2c(=O)sc3c(Cl)nc(-c4cccc([R2])c4)nc32)cc1</smiles>

Scheme 1 (i) $\mathrm{LiOH}$; (ii) $\mathrm{POCl}_{3} / \mathrm{PCl}_{5}$; (iii) $\left(\mathrm{C}_{2} \mathrm{H}_{5}\right)_{2} \mathrm{SO}_{4}$; (iiii) $\mathrm{H}_{2} \mathrm{O}$

effective against the last one. This limited data indicate that the replacement of the 7-oxo group with the small, nonpolar chloro substituent substantially increased anticancer activity. Remarkable low growth percent values against a minimum number of cell lines (mean growth) was obtained only for compound 5a which was approved for the further screening test to evaluate the growth inhibition (GI), and cytostatic and cytotoxic effects. The selected compound was additionally evaluated at tenfold dilution of five different concentrations, from $10^{-4}$ to $10^{-8} \mathrm{M}$ on approximately 60 human tumor cell lines panels. Three different dose-response parameters, $\mathrm{GI}_{50}$, TGI, and $\mathrm{LC}_{50}$, were calculated for each cell line. $\mathrm{GI}_{50}$ is the molar concentration of the compound required for half GI. Total growth inhibition (TGI) is the molar concentration of the compound resulting in TGI; TGI signifies the cytostatic effect. $\mathrm{LC}_{50}$ is the molar concentration of the compound resulting in a $50 \%$ death of the initial cells; $\mathrm{LC}_{50}$ signifies the cytotoxic effect. The overview of these parameters of compound 5a is reported in Table 4 and compared with log $\mathrm{GI}_{50}$ values of thioguanine (TG), the NCI standard anticancer agent. The $\log \mathrm{GI}_{50}$ values lower than -5 showed a notable activity level. It can be noticed that compound $\mathbf{5 a}$ proved to be very sensitive toward non-small cell lung cancer NCI-H522 and renal cancer UO-31 $\log \mathrm{GI}_{50}-5.91$ and -5.88 , respectively, (MG_MID: $\log \mathrm{GI}_{50}-5.1, \log$ TGI $\left.-4.4, \log \mathrm{LC}_{50}-4.09\right)$. GI of most cell lines of standard TG is higher than that showed by investigated compound 5a; but against the following cell lines: K-562, NCI-H322M, NCI-H522, SW-620, U251, SK-MEL-28, IGROV1, A498, and HS 578T, compound 5a was more active than TG. TG is a guanine analog and thiazolo[4,5$d$ ]pyrimidines can be considered as 7-thio analogs of the purine bases guanine and adenine. Thiazolo[4,5- $d]$ pyrimidine derivatives may interfere with the synthesis of guanine nucleotides as antimetabolites. 
Table $1{ }^{13} \mathrm{C}-\mathrm{NMR}$ data of compounds $\mathbf{5 a}, \mathbf{5 b}$, and $\mathbf{5 d}$

Comp. $\quad{ }^{13} \mathrm{C}-\mathrm{NMR}\left(\mathrm{DMSO}-\mathrm{d}_{6}\right) \delta \mathrm{ppm}$<smiles>[R]c1ccc(-c2nc(Cl)c3sc(=O)n(-c4ccccc4)c3n2)cc1</smiles>

5a R2=H 168.25 (C15), 166.79 (C5), 160.99 (C7), 157.65 (C17), 150.71 (C4), 135.08 (C6), 133.55 (C16), 133.17 (C1), 132.04 (C10), 131.69 (C13), 129.44 (C9), 129.28 (C11), 129.04 (C2), 128.94 (C3), 128.86 (C12), 128.70 (C14), 128.05 (C8)

5b R2=Cl 168.21 (C15), 166.73 (C5), 159.96 (C17), 157.67 (C7), 155.87 (C4), 150.71 (C6), 136.87 (C16), 136.54 (C1), 133.96 (C10), 133.52 (C3), 133.11 (C12), 130.66 (C13), 129.34 (C9), 129.07 (C14), 129.03 (C8), 128.93 (C11), 128.81 (C2)

5d R2=F 168.21 (C15), 166.75 (C5), 160.04 (C1), 157.59 (C17), 155.64 (C7), 150.71 (C4), 133.49 (C6), 133.11 (C16),

131.60 (C10), 130.50 (C3), 130.38 (C12), 130.19 (C9), 130.07 (C14), 129.16 (C8), 129.30 (C13), 115.97 (C2), 115.76 (C11)

The carbon atom-numbering scheme used in the crystallographic analysis was applied

Table 2 Crystallographic data for compound $\mathbf{5 a}$

\begin{tabular}{|c|c|}
\hline \multicolumn{2}{|c|}{ Crystal data and structure refinement } \\
\hline Empirical formula & $\mathrm{C}_{17} \mathrm{H}_{10} \mathrm{ClN}_{3} \mathrm{O}_{2} \mathrm{~S}$ \\
\hline Formula weight & 339.79 \\
\hline Temperature & $100(2) \mathrm{K}$ \\
\hline Wavelength & $0.71073 \AA$ \\
\hline Crystal system, space group & Monoclinic, Cc \\
\hline Unit cell dimensions & $\begin{array}{l}a=11.7588(8) \AA \alpha=90 \\
b=19.4837(14) \AA \beta=90 \\
c=7.0758(5) \AA \gamma=90\end{array}$ \\
\hline Volume & $1468.89(18) \AA^{3}$ \\
\hline Z, calculated density & $4,1.536 \mathrm{Mg} / \mathrm{m}^{3}$ \\
\hline Absorption coefficient & $0.409 \mathrm{~mm}^{-1}$ \\
\hline$F(000)$ & 696 \\
\hline Crystal size & $0.20 \times 0.10 \times 0.10 \mathrm{~mm}$ \\
\hline Theta range for data collection & $2.18-27.07$ \\
\hline Limiting indices & $-15 \Leftarrow \mathrm{h} \Leftarrow 15,-24 \Leftarrow \mathrm{k} \Leftarrow 24,-9 \Leftarrow 1 \Leftarrow 9$ \\
\hline Reflection collected/unique & $61,281 / 3,225[R$ (int) $=0.0320]$ \\
\hline Completeness to theta $=27.07$ & $99.9 \%$ \\
\hline Absorption correction & Semi-empirical from equivalents \\
\hline Max. and min transmission & 0.9602 and 0.9226 \\
\hline Refinement method & Full-matrix least-squares on $F^{2}$ \\
\hline Data/restraints/parameters & $3,225 / 3 / 208$ \\
\hline Goodness-of-fit on $F^{2}$ & 1.036 \\
\hline Final $\mathrm{R}$ indices $[I>2 \operatorname{sigma}(\mathrm{I})]$ & $R_{1}=0.0195, w R_{2}=0.0520$ \\
\hline $\mathrm{R}$ indices (all data) & $R_{1}=0.0197, w \mathrm{R}_{2}=0.0524$ \\
\hline Absolute structure parameter & $-0.02(3)$ \\
\hline Largest diff. peak and hole & 0.202 and -0.265 e. $\AA^{3}$ \\
\hline
\end{tabular}


Table 3 Anticancer activity as growth $\%$ in concentration $10^{-5} \mathrm{M}$ for the compounds $\mathbf{5 a}, \mathbf{5 b}$, and $\mathbf{5 d}$

\begin{tabular}{|c|c|c|c|}
\hline Compound & $\begin{array}{l}\text { Mean } \\
\text { growth\% }\end{array}$ & $\begin{array}{l}\text { Range of } \\
\text { growth\% }\end{array}$ & Most sensitive panel/cell line growth \% \\
\hline 5a & 71.26 & $\begin{array}{l}-84.63 \text { to } \\
124.07\end{array}$ & $\begin{array}{l}-84.63 \mathrm{Rc} / \mathrm{UO}-31,-77.98 \mathrm{M} / \mathrm{MALME}-3 \mathrm{M},-69.53 \text { NSCLc/NCI-H522, } 3.17 \text { Cc/HCC-2998, } 8.46 \mathrm{Cc} / \\
\text { HCC-116, 16.05 M/LOX IMVI, 19.57 L/CCRF-CEM, 26.33 L/SR, 33.32 Oc/OVCAR-3 }\end{array}$ \\
\hline $5 \mathbf{b}$ & 86.17 & $\begin{array}{l}5.19 \text { to } \\
136.81\end{array}$ & $\begin{array}{l}\text { 5.19 NSCLc/NCI-H522, 21.51 L/SR,24.35 M/LOX IMVI, 29.34 Cc/HCT-116, } 33.63 \text { L/CCRF-CEM, } \\
34.56 \text { L/K-562, 47.57 Cc/SW-620 }\end{array}$ \\
\hline 5d & 91.21 & $\begin{array}{c}-31.63 \text { to } \\
124.32\end{array}$ & -31.63 NSCLc/NCI-H522, 28.57 L/SR, 35.79 L/K-562, 40.46 Cc/HCT-116, 41.76 Rc/CAKI-1 \\
\hline
\end{tabular}

Data obtained from the NCIs in vitro disease-oriented human tumor cells

$L$ leukemia, $N S C L c$ non-small cell lung cancer, $C c$ colon cancer, $M$ melanoma, $O c$ ovarian cancer, $R c$ renal cancer

\section{Experimental}

\section{Chemistry}

Melting points were determined on a Boethius apparatus and were uncorrected. Elemental analyses for the synthesized compounds were performed on a Perkin Elmer 2400 (Waltham, MA, USA) analyzer, and results within $\pm 0.4 \%$ of the theoretical values were obtained for the new compounds. ${ }^{1} \mathrm{H}-\mathrm{NMR}$ and ${ }^{13} \mathrm{C}-\mathrm{NMR}$ spectra were acquired in $d_{6}$-DMSO on a Bruker ARX $300 \mathrm{MHz}$ (Bruker Analytic, Karlsruhe, Germany; Bruker AG, Fallanden, Switzerland) instrument. Tetramethylsilane was used as the internal standard and all chemical shift values were expressed in parts per million $(\delta, \mathrm{ppm})$. IR spectra were recorded on a Specord M80 spectrometer using $\mathrm{KBr}$ pellets. X-Ray Crystallography: the data were collected using the Bruker KAPPA APEXII ULTRA controlled by APEXII software. Reaction progress and the purity of the obtained compounds were monitored by thin-layer chromatography on Merck silica gel plates (Merck $F_{254}$, Darmstadt, Germany) using the solvent system dichloromethane: 1-propanol (10:1) for elution. Iodine was used as a developing agent. The chemicals and reagents for syntheses were obtained from Alfa Aesar (Karlsruhe, Germany), Chempur (Piekary Sl. Poland), and Sigma-Aldrich (Steinheim, Germany). Starting compounds are synthesized according to the literature (Gewald et al., 1966; Becan and Wagner, 2008).

\section{General procedures for the synthesis of compounds $\mathbf{4 a}-\mathbf{4 f}$} and $\mathbf{5 a}-\mathbf{5 f}$

To a solution of appropriate compound $\mathbf{2}$ or $\mathbf{3}(10 \mathrm{mmol})$ in acetonitrile $(20 \mathrm{ml})$, diethyl sulfate $(4.62 \mathrm{~g}, 30 \mathrm{mmol})$ was added, and the reaction mixture was heated under reflux for $1 \mathrm{~h}$ at $130{ }^{\circ} \mathrm{C}$. After cooling, $100 \mathrm{ml}$ of water was added and the reaction mixture was refluxed with stirring for $2 \mathrm{~h}$ during which the product was precipitated. The solid was filtered and suspended in a hot mixture of methanol and $5 \% \mathrm{NaHCO}_{3}$. The reaction mixture was allowed to cool, and the crude product was filtered and crystallized from appropriate solvent.

\section{3,5-Diphenyl-6H-thiazolo[4,5-d]pyrimidine-2,7-dione (4a)}

IR $(\mathrm{KBr}) \mathrm{cm}^{-1}:$ 3450, $3080(\mathrm{NH}), 1680(\mathrm{C}=\mathrm{O}), 1530$ $(\mathrm{C}=\mathrm{N}), 1260(\mathrm{C}-\mathrm{S}-\mathrm{C}), 760$ (phenyl). ${ }^{1} \mathrm{H}-\mathrm{NMR}\left(d_{6}\right.$-DMSO) $\delta$ : 7.42-7.93 (m, 10H, arom.), 13.19 (s, 1H, NH). Anal. Calcd for $\mathrm{C}_{17} \mathrm{H}_{11} \mathrm{~N}_{3} \mathrm{O}_{2} \mathrm{~S}$ : C, 63.54; H, 3.45; N, 13.08 . Found: C, 63.44; H, 3.52; N, 13.27.

5-(4-Chlorophenyl)-3-phenyl-6H-thiazolo[4, 5-d]pyrimidine-2,7-dione (4b)

IR $(\mathrm{KBr}) \mathrm{cm}^{-1}:$ 3450, $3090(\mathrm{NH}), 1670(\mathrm{C}=\mathrm{O}), 1590$ $(\mathrm{C}=\mathrm{N}), 1230(\mathrm{C}-\mathrm{S}-\mathrm{C}), 760$ (phenyl). ${ }^{1} \mathrm{H}-\mathrm{NMR}\left(d_{6-}\right.$ DMSO) $\delta$ : 7.51-7.94 (m, 9H, arom.), $13.22(\mathrm{~s}, 1 \mathrm{H}, \mathrm{NH})$. Anal. Calcd for $\mathrm{C}_{17} \mathrm{H}_{10} \mathrm{ClN}_{3} \mathrm{O}_{2} \mathrm{~S}$ : C, 57.39; H, 2.83; N, 11.81 . Found: C, 57.56; H, 3.01; N, 11.97.

\section{5-(2-Chlorophenyl)-3-phenyl-6H-thiazolo[4, 5-d]pyrimidine-2,7-dione $(\mathbf{4 c})$}

IR $(\mathrm{KBr}) \mathrm{cm}^{-1}: 3470,3080(\mathrm{NH}), 1680(\mathrm{C}=\mathrm{O}), 1590$ $(\mathrm{C}=\mathrm{N}), 1260(\mathrm{C}-\mathrm{S}-\mathrm{C}), 760$ (phenyl). ${ }^{1} \mathrm{H}-\mathrm{NMR}\left(d_{6}\right.$-DMSO) $\delta$ : 7.34-7.99 (m, 9H, arom.), 13.27 (s, 1H, NH). Anal. Calcd for $\mathrm{C}_{17} \mathrm{H}_{10} \mathrm{ClN}_{3} \mathrm{O}_{2} \mathrm{~S}$ : C, 57.39; H, 2.83; N, 11.81 . Found: C, 57.59; H, 2.87; N, 11.85.

5-(4-Fluorophenyl)-3-phenyl-6H-thiazolo[4, 5-d]pyrimidine-2,7-dione (4d)

IR $(\mathrm{KBr}) \mathrm{cm}^{-1}:$ 3450, $3090(\mathrm{NH}), 1680(\mathrm{C}=\mathrm{O}), 1610$ $(\mathrm{C}=\mathrm{N}), 1240$ (C-S-C), 770 (phenyl). ${ }^{1} \mathrm{H}-\mathrm{NMR}\left(d_{6}\right.$-DMSO) $\delta$ : 7.31-8.20 (m, 9H, arom.), $13.20(\mathrm{~s}, 1 \mathrm{H}, \mathrm{NH})$. Anal. 
Table 4 The result of the in vitro anticancer activity of compound $\mathbf{5 a}$ against 60 human cancer cell lines

\begin{tabular}{|c|c|c|c|c|c|}
\hline \multirow[t]{2}{*}{ Panel Cell line } & \multicolumn{4}{|l|}{ Compound 5a } & \multirow{2}{*}{$\begin{array}{l}\text { TG } \\
\log \mathrm{Gl}_{50}\end{array}$} \\
\hline & Growth $(\%)^{\mathrm{b}} 10^{-5} \mathrm{M}$ & $\log \mathrm{Gl}_{50}$ & Log TGI & $\log \mathrm{LC}_{50}$ & \\
\hline \multicolumn{6}{|l|}{ Leukemia } \\
\hline CCRF-CEM & 23 & -5.42 & $-^{\mathrm{c}}$ & - & -6.5 \\
\hline HL-60(TB) & 38 & -5.23 & - & - & $\mathrm{ns}$ \\
\hline $\mathrm{K}-562$ & 19 & -5.39 & - & - & -4.0 \\
\hline MOLT-4 & 54 & -4.89 & - & - & $n s^{\mathrm{d}}$ \\
\hline RPMI-8226 & 75 & -4.35 & - & - & -5.9 \\
\hline SR & 8 & -5.58 & -4.67 & - & -6.2 \\
\hline \multicolumn{6}{|l|}{ Non-Small } \\
\hline \multicolumn{6}{|l|}{ Cell Lung $C$. } \\
\hline A549/ATCC & 87 & -4.36 & - & - & -5.3 \\
\hline EKVX & 84 & -4.59 & - & - & -5.4 \\
\hline HOP-62 & 15 & -5.44 & -4.81 & -4.14 & -6.1 \\
\hline HOP-92 & 24 & -5.51 & -4.17 & - & -5.8 \\
\hline NCI-H23 & 20 & -5.44 & -4.51 & - & -5.5 \\
\hline NCI-H322 M & 62 & -4.85 & -4.23 & - & -4.6 \\
\hline NCI-H460 & 78 & -4.60 & - & - & -6.0 \\
\hline NCI-H522 & -65 & -5.91 & -5.52 & - & -5.7 \\
\hline \multicolumn{6}{|l|}{ Colon $C$. } \\
\hline COLO-205 & 52 & -4.95 & - & - & -5.6 \\
\hline HCC-2998 & 90 & -4.09 & - & - & $\mathrm{ns}$ \\
\hline HCT-116 & -53 & -5.68 & -5.35 & -5.02 & -6.2 \\
\hline НCТ-15 & 28 & -5.33 & - & - & -5.6 \\
\hline HT29 & 10 & -5.41 & -4.72 & - & -5.9 \\
\hline KM12 & 81 & -4.09 & - & - & -5.5 \\
\hline SW620 & -4 & -5.56 & -5.04 & - & -5.4 \\
\hline \multicolumn{6}{|l|}{ CNS Cancer } \\
\hline SF-268 & 52 & -4.98 & -4.42 & - & -5.9 \\
\hline SF-295 & 92 & -4.24 & - & - & -5.9 \\
\hline SF-539 & 52 & -4.96 & - & - & -6.2 \\
\hline SNB-19 & 70 & -4.38 & - & - & -4.1 \\
\hline SNB-75 & 12 & -5.73 & -4.86 & -4.25 & -6.0 \\
\hline U251 & 20 & -5.43 & -4.73 & - & -5.0 \\
\hline \multicolumn{6}{|l|}{ Melanoma } \\
\hline LOX IMVI & -44 & -5.69 & -5.32 & -4.74 & ns \\
\hline MALME-3M & 62 & -4.83 & -4.10 & - & -5.5 \\
\hline M14 & 16 & -5.42 & -4.45 & - & -6.2 \\
\hline MDA-MB-435 & 26 & -5.31 & -4.34 & - & -6.3 \\
\hline SK-MEL-2 & 48 & -5.04 & -4.36 & - & -5.8 \\
\hline SK-MEL-28 & 9 & -5.47 & -4.88 & -4.16 & -5.2 \\
\hline SK-MEL-5 & 60 & -4.81 & - & - & -5.6 \\
\hline UACC-257 & 48 & -5.05 & -4.50 & - & -5.2 \\
\hline UACC-62 & 62 & -4.70 & - & - & -6.4 \\
\hline \multicolumn{6}{|l|}{ Ovarian $C}$. \\
\hline IGROV1 & -65 & -5.75 & -5.32 & -4.74 & -5.2 \\
\hline OVCAR-3 & -41 & -5.75 & -4.10 & - & -5.8 \\
\hline OVCAR-4 & 31 & -5.30 & -4.45 & - & -5.3 \\
\hline OVCAR-5 & 90 & - & -4.34 & - & -6.3 \\
\hline
\end{tabular}


Table 4 continued

\begin{tabular}{|c|c|c|c|c|c|}
\hline \multirow[t]{2}{*}{ Panel Cell line } & \multicolumn{4}{|l|}{ Compound $\mathbf{5 a}$} & \multirow{2}{*}{$\begin{array}{l}\text { TG } \\
\log \mathrm{Gl}_{50}\end{array}$} \\
\hline & Growth $(\%)^{\mathrm{b}} 10^{-5} \mathrm{M}$ & $\log \mathrm{Gl}_{50}$ & Log TGI & $\log \mathrm{LC}_{50}$ & \\
\hline OVCAR-8 & -45 & -5.69 & -4.36 & - & -6.4 \\
\hline NCI/ADR-RES & 66 & -4.67 & - & - & -6.4 \\
\hline SK-OV-3 & 81 & - & - & - & -6.3 \\
\hline \multicolumn{6}{|l|}{ Renal Cancer } \\
\hline $786-0$ & 41 & -5.15 & -4.25 & - & -5.8 \\
\hline A498 & 44 & -5.46 & - & - & -4.6 \\
\hline $\mathrm{ACHN}$ & 42 & -5.16 & - & - & -5.4 \\
\hline CAKI-1 & -30 & -5.63 & -5.24 & -4.33 & -6.5 \\
\hline SN12C & 43 & -5.13 & - & - & -5.1 \\
\hline TK-10 & 51 & -4.98 & - & - & -6.3 \\
\hline UO-31 & -79 & -5.88 & -5.54 & - & -6.1 \\
\hline RXF 393 & -4 & -5.62 & -5.05 & -4.42 & -6.3 \\
\hline \multicolumn{6}{|l|}{ Prostate $C$. } \\
\hline PC-3 & 11 & -5.48 & -4.84 & -4.09 & -5.5 \\
\hline DU-145 & 34 & -5.33 & -4.63 & -4.09 & -6.3 \\
\hline \multicolumn{6}{|l|}{ Breast $C$. } \\
\hline MCF7 & 77 & -4.19 & - & - & -6.3 \\
\hline MDA-MB-231/ATCC & 37 & -5.20 & - & - & ns \\
\hline HS 578T & 12 & -5.48 & -4.73 & - & -5.2 \\
\hline ВТ-549 & 86 & - & - & - & -5.9 \\
\hline $\mathrm{T}-47 \mathrm{D}$ & 57 & -4.77 & - & - & -5.0 \\
\hline MDA-MB-468 & 20 & -5.44 & - & - & $\mathrm{ns}$ \\
\hline MG_MID ${ }^{\mathrm{e}}$ & & -5.1 & -4.4 & -4.09 & \\
\hline
\end{tabular}

${ }^{a}$ Data obtained from the NCI's in vitro disease-oriented human tumor cells

b Values greater than zero mean percentage of growth and those less than zero mean percentage of lethality to the tumor cell line

c The values greater than -4 were excluded

${ }^{\mathrm{d}}$ Cell line not screened

e MG_MID (mean graph midpoint) arithmetical mean value for all tested cell lines

Calcd for $\mathrm{C}_{17} \mathrm{H}_{10} \mathrm{FN}_{3} \mathrm{O}_{2} \mathrm{~S}: \mathrm{C}, 60.17 ; \mathrm{H}, 2.97 ; \mathrm{N}, 12.38$. Found: C, 59.98; H, 3.03; N, 12.41 .

\section{3,5-Bis(4-fluorophenyl)-6H-thiazolo[4,5-d]pyrimidine-2, 7-dione $(4 \mathrm{e})$}

IR (KBr) cm $\mathrm{cm}^{-1}: 3470,3090(\mathrm{NH}), 1690(\mathrm{C}=\mathrm{O}), 1570$ $(\mathrm{C}=\mathrm{N}), 1240(\mathrm{C}-\mathrm{S}-\mathrm{C}), 780$ (phenyl). ${ }^{1} \mathrm{H}-\mathrm{NMR}\left(d_{6}\right.$-DMSO) $\delta$ : 7.22-8.03 (m, 8H, arom.), $13.21(\mathrm{~s}, 1 \mathrm{H}, \mathrm{NH})$. Anal. Calcd for $\mathrm{C}_{17} \mathrm{H}_{9} \mathrm{FN}_{3} \mathrm{O}_{2} \mathrm{~S}: \mathrm{C}, 57.14 ; \mathrm{H}, 2.54 ; \mathrm{N}, 11.76$. Found: C, 57.31; H, 2.55; N, 11.94 .

3-(4-Bromophenyl)-5-phenyl-6H-thiazolo[4,

5-d]pyrimidine-2,7-dione (4f)

IR (KBr) cm $\mathrm{cm}^{-1}: 3450,3080(\mathrm{NH}), 1680(\mathrm{C}=\mathrm{O}), 1590$ $(\mathrm{C}=\mathrm{N}), 1260(\mathrm{C}-\mathrm{S}-\mathrm{C}), 760$ (phenyl). ${ }^{1} \mathrm{H}-\mathrm{NMR}\left(d_{6}\right.$-DMSO) $\delta$ : 7.45-8.16 (m, 9H, arom.), 13.19 (s, 1H, NH). Anal.
Calcd for $\mathrm{C}_{17} \mathrm{H}_{10} \mathrm{BrN}_{3} \mathrm{O}_{2} \mathrm{~S}$ : C, 51.01; H, 2.52; N, 10.50 . Found: C, 51.14; H, 2.60; N, 10.66 .

\section{7-Chloro-3,5-diphenyl-thiazolo[4,5-d]pyrimidin-2-one (5a)}

IR $(\mathrm{KBr}) \mathrm{cm}^{-1}: 1680(\mathrm{C}=\mathrm{O}), 1590(\mathrm{C}=\mathrm{N}), 1260(\mathrm{C}-\mathrm{S}-\mathrm{C})$, 760 (phenyl). ${ }^{1} \mathrm{H}-\mathrm{NMR}\left(d_{6}\right.$-DMSO) $\delta: 7.46-8.13(\mathrm{~m}, 10 \mathrm{H}$, arom.). Anal. Calcd for $\mathrm{C}_{17} \mathrm{H}_{10} \mathrm{ClN}_{3} \mathrm{OS}: \mathrm{C}, 60.09 ; \mathrm{H}, 2.97$; N, 12.37. Found: C, 59.98; H, 3.01; N, 12.38 .

7-Chloro-5-(4-chlorophenyl)-3-phenyl-thiazolo[4, 5-d]pyrimidin-2-one $(\mathbf{5 b})$

IR $(\mathrm{KBr}) \mathrm{cm}^{-1}: 1680(\mathrm{C}=\mathrm{O}), 1560(\mathrm{C}=\mathrm{N}), 1230(\mathrm{C}-\mathrm{S}-\mathrm{C})$, 760 (phenyl). ${ }^{1} \mathrm{H}-\mathrm{NMR}\left(d_{6}\right.$-DMSO) $\delta: 7.52-8.11(\mathrm{~m}, 9 \mathrm{H}$, arom.). Anal. Calcd for $\mathrm{C}_{17} \mathrm{H}_{9} \mathrm{Cl}_{2} \mathrm{~N}_{3} \mathrm{OS}$ : C, 54.56; $\mathrm{H}, 2.42$; $\mathrm{N}, 11.23$. Found: C, 54.60; H, 2.49; N, 11.29. 
Table 5 Characterization data of compounds $\mathbf{4 a}-\mathbf{4 f}$ and $\mathbf{5 a}-\mathbf{5 f}$

\begin{tabular}{llllllll}
\hline Compound & $\mathrm{R} 1$ & $\mathrm{R} 2$ & $\mathrm{~m} . \mathrm{p} .\left({ }^{\circ} \mathrm{C}\right)$ & Yield $\%$ & Molecular formula & Molecular weight & Solvent \\
\hline 4a & - & - & $279-280$ & 64.55 & $\mathrm{C}_{17} \mathrm{H}_{11} \mathrm{~N}_{3} \mathrm{O}_{2} \mathrm{~S}$ & 321,35 & 1 -Propanol \\
4b & - & $4-\mathrm{Cl}$ & $369-370$ & 58.77 & $\mathrm{C}_{17} \mathrm{H}_{10} \mathrm{ClN}_{3} \mathrm{O}_{2} \mathrm{~S}$ & 355,81 & DMF-1-propanol 3:1 \\
$\mathbf{4 c}$ & - & $2-\mathrm{Cl}$ & $338-339$ & 58.88 & $\mathrm{C}_{17} \mathrm{H}_{10} \mathrm{ClN}_{3} \mathrm{O}_{2} \mathrm{~S}$ & 355,81 & DMF-1-propanol 3:1 \\
4d & - & $4-\mathrm{F}$ & $348-349$ & 56.51 & $\mathrm{C}_{17} \mathrm{H}_{10} \mathrm{FN}_{3} \mathrm{O}_{2} \mathrm{~S}$ & 339,31 & DMF-water \\
4e & $4-\mathrm{F}$ & $4-\mathrm{F}$ & $327-328$ & 49.73 & $\mathrm{C}_{17} \mathrm{H}_{9} \mathrm{~F}_{2} \mathrm{~N}_{3} \mathrm{O}_{2} \mathrm{~S}$ & 357,33 & $1-\mathrm{Propanol}$ \\
$\mathbf{4 f}$ & $4-\mathrm{Br}$ & - & $210-211$ & 55.77 & $\mathrm{C}_{17} \mathrm{H}_{10} \mathrm{BrN}_{3} \mathrm{O}_{2} \mathrm{~S}$ & 400,25 & DMF-1-propanol 3:1 \\
$\mathbf{5 a}$ & - & - & $194-195$ & 63.87 & $\mathrm{C}_{17} \mathrm{H}_{10} \mathrm{ClN}_{3} \mathrm{OS}$ & 339,80 & DMF-1-propanol 3:1 \\
5b & - & $4-\mathrm{Cl}$ & $171-172$ & 57.45 & $\mathrm{C}_{17} \mathrm{H}_{9} \mathrm{Cl}_{2} \mathrm{~N}_{3} \mathrm{OS}$ & 374,24 & DMF-water \\
5c & - & $2-\mathrm{Cl}$ & $240-241$ & 52.32 & $\mathrm{C}_{17} \mathrm{H}_{9} \mathrm{Cl}_{2} \mathrm{~N}_{3} \mathrm{OS}$ & 374,24 & DMF-1-propanol 3:1 \\
5d & - & $4-\mathrm{F}$ & $235-236$ & 54.73 & $\mathrm{C}_{17} \mathrm{H}_{9} \mathrm{ClFN}_{3} \mathrm{OS}$ & 357,79 & DMF-1-propanol 3:1 \\
5e & $4-\mathrm{F}$ & $4-\mathrm{F}$ & $234-235$ & 48.67 & $\mathrm{C}_{17} \mathrm{H}_{8} \mathrm{ClF}_{2} \mathrm{~N}_{3} \mathrm{OS}$ & 375,78 & DMF-1-propanol 3:1 \\
5f & $4-\mathrm{Br}$ & - & $388-390$ & 57.46 & $\mathrm{C}_{17} \mathrm{H}_{9} \mathrm{BrClN}_{3} \mathrm{OS}$ & 418,69 & DMF-1-propanol 3:1 \\
\hline
\end{tabular}

\section{7-Chloro-5-(2-chlorophenyl)-3-phenyl-thiazolo [4,5-d]pyrimidin-2-one $(\mathbf{5 c})$}

IR $(\mathrm{KBr}) \mathrm{cm}^{-1}: 1690(\mathrm{C}=\mathrm{O}), 1570(\mathrm{C}=\mathrm{N}), 1250(\mathrm{C}-\mathrm{S}-\mathrm{C})$, 760 (phenyl). ${ }^{1} \mathrm{H}-\mathrm{NMR}\left(d_{6}\right.$-DMSO) $\delta: 7.52-8.11$ (m, 9H, arom.). Anal. Calcd for $\mathrm{C}_{17} \mathrm{H}_{9} \mathrm{Cl}_{2} \mathrm{~N}_{3} \mathrm{OS}$ : C, 54.56; $\mathrm{H}, 2.42$; $\mathrm{N}, 11.23$. Found: C, 54.65; H, 2.50; N, 11.33 .

\section{7-Chloro-5-(4-fluorophenyl)-3-phenyl-thiazolo [4,5-d]pyrimidin-2-one $(\mathbf{5 d})$}

IR (KBr) cm ${ }^{-1}: 1690(\mathrm{C}=\mathrm{O}), 1600(\mathrm{C}=\mathrm{N}), 1240(\mathrm{C}-\mathrm{S}-\mathrm{C})$, 760 (phenyl). ${ }^{1} \mathrm{H}-\mathrm{NMR}\left(d_{6}\right.$-DMSO) $\delta: 7.27-8.15(\mathrm{~m}, 9 \mathrm{H}$, arom.). Anal. Calcd for $\mathrm{C}_{17} \mathrm{H}_{9} \mathrm{ClN}_{3} \mathrm{OS}$ : C, 57.10; H, 2.73; N, 11.75. Found: C, 57.21; H, 2.86; N, 11.83 .

\section{7-Chloro-3,5-bis(4-fluorophenyl)thiazolo[4,5-d]pyrimidin- 2-one $(5 \mathrm{e})$}

IR $(\mathrm{KBr}) \mathrm{cm}^{-1}: 1690(\mathrm{C}=\mathrm{O}), 1590(\mathrm{C}=\mathrm{N}), 1250(\mathrm{C}-\mathrm{S}-$ C), 770 (phenyl). ${ }^{1} \mathrm{H}-\mathrm{NMR}\left(d_{6}\right.$-DMSO) $\delta: 7.26-8.22(\mathrm{~m}$, $8 \mathrm{H}$, arom.). Anal. Calcd for $\mathrm{C}_{17} \mathrm{H}_{8} \mathrm{ClF}_{2} \mathrm{~N}_{3} \mathrm{OS}$ : C, 54.34; $\mathrm{H}$, 2.15; N, 11.18. Found: C, 54.42; H, 2.20; N, 11.26.

\section{3-(4-Bromophenyl)-7-chloro-5-phenyl-thiazolo[4,5- d]pyrimidin-2-one (5f)}

IR $(\mathrm{KBr}) \mathrm{cm}^{-1}: 1680(\mathrm{C}=\mathrm{O}), 1560(\mathrm{C}=\mathrm{N}), 1250(\mathrm{C}-\mathrm{S}-\mathrm{C})$, 770 (phenyl). ${ }^{1} \mathrm{H}-\mathrm{NMR}\left(d_{6}\right.$-DMSO) $\delta: 7.33-8.16(\mathrm{~m}, 8 \mathrm{H}$, arom.). Anal. Calcd for $\mathrm{C}_{17} \mathrm{H}_{9} \mathrm{BrClN}_{3} \mathrm{OS}: \mathrm{C}, 48.77 ; \mathrm{H}$, 2.17; N, 10.04. Found: C, 48.91; H, 2.25; N, 10.12 .

Characteristic data of the new compounds are depicted in Table 5.

\section{Antitumor in vitro screening}

The antitumor studies were performed at the NCI (Bethesda, MD, USA). The test agents were preliminarily evaluated in the full panel of 60 different human tumor cell lines derived from nine cancer diseases, namely leukemia, melanoma, non-small cell lung c., colon c., brain c., breast c., ovarian c., prostate c., and renal c., at single $10^{-5} \mathrm{M}$ concentration. Compounds with significant GI are evaluated at five different concentrations ranging from $10^{-4}$ to $10^{-8} \mathrm{M}$. The percent growth was evaluated versus controls not treated with tested compounds. Preparation of the tested compounds and the sulforhodamine B (SRB) protein assay which was used to estimate cell viability of growth were described previously (Becan and Wagner, 2008; Monks et al., 1991; Boyd and Paull, 1995; Shoemaker et al., 2002).

Acknowledgments The authors thank the staff of the Department of Health and Human Services, National Institutes of Health (Bethesda, MD, USA), for in vitro evaluation of anticancer activity.

Open Access This article is distributed under the terms of the Creative Commons Attribution License which permits any use, distribution, and reproduction in any medium, provided the original author(s) and the source are credited.

\section{References}

Akbari JB, Mehta KB, Pathak SJ, Joshi HS (2008) Synthesis and antimicrobial activity of some new pyrazolo[3,4- $d]$ pyrimidines and thiazolo[4,5- $d$ ]pyrimidines. Indian J Chem 47B:477-480

Becan L, Wagner E (2008) Synthesis and antitumor screening of novel 3-phenylthiazolo[4,5- $d$ ]pyrimidine-2 thione derivatives. Arzneim-Forsch/Drug Res 58(10):521-528 
Beck JP, Curry MA, Chorvat RJ, Fitzgerald LW, Giligan PJ, Zaczek $\mathrm{R}$, Trainor GL (1999) Thiazolo[4,5-d]-pyrimidine thiones and ones as corticotrophin-releasing hormone (CRH-R1) receptor antagonists. Bioorg Med Chem Lett 9:1185-1188

Boyd MR, Paull KD (1995) Some practical considerations and applications of the National Cancer Institute in vitro anticancer drug discovery screen. Drug Dev Res 34:91-109

Fahmy HTY, Rostom SAF, Bekhit AA (2002) Synthesis and antitumor evaluation of new polysubstituted thiazole and derived thiazolo[4,5- $d]$ pyrimidine systems. Arch Pharm Pharm Med Chem 5:213-222

Fahmy HTY, Rostom AAF, Saudi MN, Zjawiony JK, Robins DJ (2003) Synthesis and in vitro evaluation of the anticancer activity of novel fluorinated thiazolo[4,5- $d$ ]pyrimidines. Arch Pharm Pharm Med Chem 336:216-225

Gewald K (1966) Reaktion von methylenaktiven Nitrilen mit Senfölen und Schwefel. J Prakt Chem 32:26-30

Habib N, Soliman R, El-Tombary A, El-Hawash S, Shaaban O (2007) Synthesis of thiazolo[4,5- $d$ ]- pyrimidine derivatives as potential antimicrobial agents. Arch Pharm Res 30(12):1511-1520

Monks A, Scudiero DA, Skehan P, Shoemaker RH, Paull KD, Vistica DT, Hose C, Langley J, Cronise P, Vaigro-Wolff A, Gray-Goodrich M, Cambell H, Mayo J, Boyd M (1991)
Feasibility of a high-flux anticancer drug screen using a diverse panel of cultured human tumor cell lines. J Natl Cancer Inst 83:757-776

Revankar GR, Ojwang JO, Mustain SD, Rando RF, De Clerq E, Huffman JH, Drach JC, Sommadossi JP, Lewis AF (1998) Thiazolo $[4,5-d]$ pyrimidines. Part II. Synthesis and anti-human cytomegalovirus activity in vitro of certain acyclonucleosides and acyclonucleosides derived from the guanine analogue 5-aminothiazolo[4,5- $d$ ]pyrimidine-2,7(3H,6H)-dione. Antivir Chem Chemother 9:53-63

Rida SM, Habib NS, Badawey EAM, Fahmy HTY, Ghozlan HA (1996) Synthesis of novel thiazolo[4,5- $d]$-pyrimidine derivatives for antimicrobial, anti-HIV and anticancer investigation. Pharmazie 51:927-931

Shoemaker RH, Scudiero DA, Melillo G (2002) Application of highthroughput, molecular-targeted screening to anticancer drug discovery. Curr Top Med Chem 2(3):229-246

Walters I, Austin C, Austin R, Bonnet R, Cage P, Christie J, Ebden M, Gardiner S, Grahames C, Hill S, Jewell R, Hunt F, Lewis S, Martin I, Nicholls D, Robinson D (2008) Evaluation of a series of bicyclic CXCR2 antagonists. Bioorg Med Chem Lett 18(2): 798-803 